\title{
Location-Dependent Information Extraction for Positioning
}

\author{
Marios Raspopoulos*, Benoît Denis ${ }^{\dagger}$, Mohamed Laaraiedh ${ }^{\ddagger}$, Jacobo Domínguez ${ }^{\S}$, Lorena de Celis ${ }^{\S}$, Dirk Slock ${ }^{\Uparrow}$ \\ George Agapiou**, Julien Stéphan "I and Stavros Stavrou \\ *Sigint Solutions Ltd, Nicosia, Cyprus, Email: m.raspopoulos@sigintsolutions.com \\ †CEA-Leti Minatec, Grenoble, France, Email: benoit.denis@cea.fr \\ †University of Rennes 1, Rennes, France, Email: mohamed.laaraiedh@univ-rennes1.fr \\ $\S$ Acorde Technologies S.A, Santander Spain, Email: \{jacobo.dominguez,lorena.decelis\}@acorde.com \\ I EURECOM, Sophia-Antipolis, France, Email: dirk.slock@eurecom.fr \\ \| Siradel, Rennes, France, Email: jstephan@siradel.com \\ ** OTE Telecom, Athens, Greece, Email: gagapiou@oteresearch.gr
}

\begin{abstract}
This paper presents an overview of current research investigations within the WHERE-2 Project with respect to location-dependent information extraction and how this information can be used towards the benefit of positioning. It is split into two main sections; the first one relies on non-radio means such as inertial sensors and prior knowledge about the environment geometry, which can be used in the form of map constraints to improve user positioning precision in indoor environments. The second section presents how location-specific radio information can be exploited in a more sophisticated way into advanced positioning algorithms. The intended solutions include exploitation of the slow fading dynamics in addition to the fast-fading parameters, adaptation of the system to its environment on both network and terminal sides and also how specific environmental properties such as the dielectric wall parameters can be extracted and thereafter used for more accurate fingerprinting database generation using Ray Tracing modelling methods. Most of the techniques presented herein rely on real-life measurements or experiments.
\end{abstract}

\section{INTRODUCTION}

The more information that is being considered into a position estimation problem, the higher probability of a more accurate result is. Depending on the capabilities of the terminal or overall system in storing, monitoring and extracting locationspecific radio (e.g. signal strength, angle of arrival, time of arrival, impulse responses) or non-radio parameters (inertial measurements, prior map/layout knowledge), advanced positioning algorithms can be developed which may fuse the extracted radio context to provide improved positioning precision. However, specific radio parameters used in the estimation of the position many times may impose various limits on the localisation accuracy that might be hard to be exceeded. Therefore, by considering also non-radio means (information or parameters) such as inertial sensors or any prior knowledge regarding the map/layout of the environment of the users to be positioned, new solutions may put forward enabling data fusion or matching which would potentially improve the localisation accuracy.

Inertial Navigation Systems (INSs) combining different kinds of sensors (e.g. accelerometers, magnetometers, and gyroscopes) have proved to adequately complement existing navigation means such as GPS/GNSS. The knowledge of the mobility pattern (velocity, orientation, direction) of the device can be processed in order to predict or even assign higher probabilities to future immediate positions of the portable device, allowing better positioning precision or even constitute the primary positioning system when in some areas the terminal might be out of the radio coverage of the base stations or access points.

The information from inertial sensors can be used in conjunction with environment maps to improve the precision of the position prediction. The idea is to utilise the environment description availability (building databases, blueprints of indoor areas, satellite photography) for the purpose of aiding the user localisation process. By using map information, the movement of the user can be constrained and/or different probabilities can be assigned to different areas of the environment where the user might reside.

Besides non-radio assisting means, advanced positioning techniques which utilise the available radio context monitored and extracted by the terminal or by the base stations are considered for improved estimation precision. These involve (1) location through the tracking of dynamic multipath components, (2) Adaptation to the environment and (3) Modelling and estimation of environment related parameters from the radio channel response.

Section II describes how non-radio aided context is extracted and used for positioning whereas Section III deals with the exploitation of radio-aided context into improved positioning techniques.

\section{Non-RAdio Aided Positioning}

\section{A. Exploiting Inertial Sensors}

Inertial navigation is a self-contained navigation technique in which inertial sensors measurements are used to track the position and orientation of an object relative to a known starting point, orientation and velocity. An Inertial Measurement Unit (IMU) comprises accelerometers and gyroscopes and measures and reports velocity, orientation and gravitational forces and reports these to a computer, which calculates 
its current position based on velocity and time. An attitude heading reference system, AHRS, consists of sensors, gyroscopes, accelerometers and magnetometers on all three axes that provide heading, attitude and yaw information. The key difference between an IMU and an AHRS is the addition of an on-board processing system in an AHRS which provides solved attitude and heading solutions versus an IMU which just delivers sensor data to an additional device that solves the attitude solution. The inclusion of inertial sensor information into positioning is being studied and will be tested through a heterogeneous test-bed, with different wireless technologies (UWB, Zigbee, LTE).

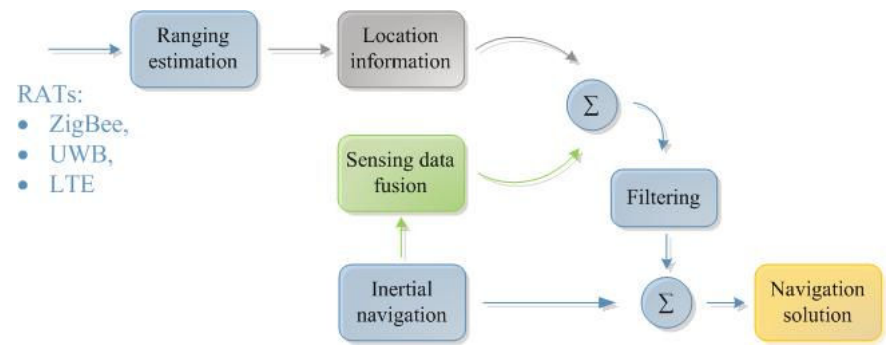

Fig. 1. Integrated navigation system architecture

In Figure 1 a data flow of the combination of inertial context with position estimation is presented. Once the inertial information is obtained, it is filtered or fused with preliminary position estimation or any other location information to improve the final position estimation. Apart from performance considerations, this fusion assumes a more relevant role, since future personal terminals are expected to integrate multiple standards and sensors, hence making heterogeneous modalities available on the user side.

1) Inertial Sensors usefulness for positioning: An initial inertial sensor study, a PCB implementation and a data fusion algorithm implementation have been carried out. Generally, context from these sensors are fused using a Kalman filter to obtain quantities of interest. Therefore, implementation of low computational cost Kalman filters is also being analysed. The first step towards an inertial sensor module is the sensors selection process. An initial market survey has been performed in order to evaluate commonly used sensors. A small set of sensors has been selected based mainly on their (1) Connectivity, (2) power consumption, (3) availability and (4) price. These sensors have been mounted and tested in a preliminary board on which one accelerometer (LIS331), one gyroscope (ITG-3200) and one magnetometer (HMC5883LTR) have been implemented.

Each type of sensor has its strong and weak points. The idea behind sensor data fusion is that characteristics of one type of sensor are used to overcome the limitations of another. Although Kalman filter was originally widely used, there are relatively new several implementations of IMU and AHRS sensor fusion algorithms specially suited for low cost boards. Some of these algorithms [1], [2] are currently being tested with the board implemented in order to find the best solution.
2) Kalman filtering for position tracking using IMU information: The topic addressed here is adaptive Kalman Filtering (AKF), dealing with joint estimation of the state sequence and parameters appearing in the state space model. The application is position tracking with simultaneous estimation of the acceleration dynamics and/or IMU parameters. The latest state of this research can be found in [3], which observes that the Expectation-Maximization (EM-KF) approach is among the most powerful techniques currently in use.

3) Pedestrian navigation fusing non-collocated IMUs and IR-UWB devices: Despite fine intrinsic localization capabilities, Impulse Radio - Ultra Wideband (IR-UWB) systems still suffer from harsh Non-Line of Sight (NLOS) conditions in practical indoor environments. In this context, a pedestrian tracking solution has been put forward in [4], which relies on the loose fusion of an IR-UWB handset transmitter with a shoe-mounted IMU including a 3-axis accelerometer and a 3axis gyroscope (3A3G), as shown on Figure 2-(a). A specific Extended Kalman Filter (EKF) has thus been defined to hybridize such non-collocated radio and inertial devices. This filter combines TOA-based UWB measurements from energy detectors [5] into new observations defined as Combined TDOA (CTDOA) [6]. While reducing filter complexity, the proposed solution enables to remove outlier measurements, without reconfiguring the whole tracking filter structure. Regarding inertial data, the IMU placement helps to identify stance phases and to periodically reset the foot velocity, hence mitigating classical drift effects. The obtained pedestrian heading and body velocity finally control the filter state prediction.

Real experiments were carried out in the representative indoor environment of Figure 2-(b), illustrating the complementarities of the two sub-systems. In particular, it is shown on Figure 2-(c) that the IMU part can adequately assist IRUWB in generalized NLOS situations and/or in penalizing locations where harmful geometrical configurations would be experienced with respect to the receivers (e.g. in Room B here). Overall, coupling both sub-systems enables reliable and robust tracking with uniform spatial quality of the location service. But these results also disclose perspectives in terms of parsimonious fusion to save energy/complexity, by switching on-demand from one stand-alone sub-system into the complete fusion-oriented scheme depending on the current conditions (i.e. NLOS or geometric dilution of precision).

\section{B. Map Matching}

Proper use of any available information into the localisation problem is something that might improve the positioning accuracy. In fact, information from inertial sensors in combination with environment maps offers this extra knowledge which aims to improve the positioning prediction. This section describes how this environment knowledge can be extracted and exploited and how it can be used into the localisation process using probabilistic fingerprinting techniques and a Robust Geometric Localisation Algorithm (RGPA).

1) Extraction of map constraints: The concept of map matching basically consists in employing a known environ- 

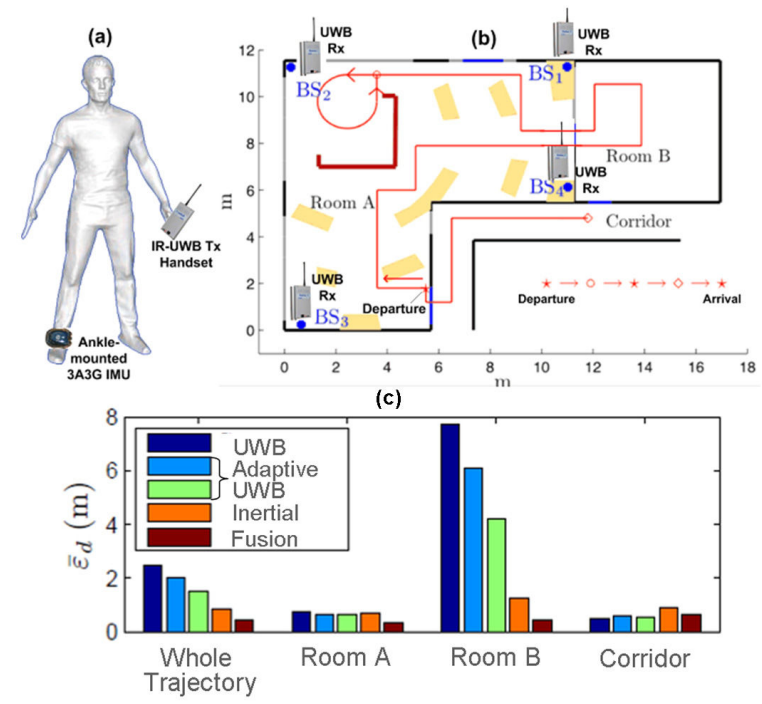

Fig. 2. (a) Pedestrian equipped with an IR-UWB transmitter handset and an ankle-mounted 3A3G IMU; (b) Real 100m-long trajectory and indoor layout, with 4 receivers (BSs) in Room A; (c) Average location error for various tracking options: IR-UWB (Deep blue), IR-UWB with adaptive observation covariance (Blue) and outliers detection (Green), IMU (Orange), IR-UWB/IMU loose fusion (Brown).

ment map for the purpose of aiding the user localization process. Recent work has notably shown significant improvements in the position prediction when environment maps are used jointly with information from inertial sensors. In indoor, the building layout usually relies on a CAD (ComputerAided Design) description of the building external walls and partitions, and can be refined with description and location of the most important pieces of furniture like cupboards, tables or desks. This digital representation is then processed to derive different kinds of map constraints either directly inferred from the geometry (simple map constraints may distinguish areas where regular users are mostly moving, static or are not expected to be located like inside wall/furniture) or by using jointly the geometry and the knowledge of the mobility of specific users or specific user classes.

2) Map Constraints into fingerprinting positioning: This approach is based on the RSS Bayesian inference method [7] which uses a probabilistic approach to perform fingerprinting positioning as it tries to find the maximum of a likelihood probability function. It takes into consideration a-priori knowledge as well as the probability of the user in being a specific location. The latter allows to incorporate map constraints into the position estimation by assigning different probabilities to specific areas of an environment describing the likelihood that the user may be located in specific areas of the environment.

For a position space $(S)$ and fingerprints $(o)$, the fingerprinting positioning algorithms that adopted tries to correlate the real-time observation $\left(o^{\prime}\right)$ with the database set $(o)$ and find the position state $\left(s_{k}\right)$ that maximises the following:

$$
P\left(s_{k} \mid o^{\prime}\right)=\frac{P\left(o^{\prime} \mid s_{k}\right) P\left(s_{k}\right)}{\sum_{k=1}^{K} P\left(o^{\prime} \mid s_{k}\right) P\left(s_{k}\right)}
$$

$P\left(o^{\prime} \mid s_{k}\right)$ is learned at the survey phase and expresses the probability for a real time measurement to be collected at a specific location (state). It requires that multiple observations for specific states are made in order to be able to assign this possibility. $P\left(s_{k}\right)$ is the probability that a user is at state $s_{k}$. If no other knowledge is used, then it is assumed that the user has equal probability of being in any position in space (therefore $\left.P\left(s_{k}\right)=1 / k\right)$. However, we plan to introduce map constraints therefore $P\left(s_{k}\right)$ will express the probability of a user being in a particular location more realistically based on the given environment. Current measurement results have indicated that for a typical indoor environment, this method achieves a positioning accuracy around $1.59 \mathrm{~m}$. Future work includes generation of map constraints as described in section II-B. 1 and investigation whether the reported accuracy of this probabilistic algorithm can be further improved.

3) Map constraints within the RGPA technique: This approach uses map constraints to feed the RGPA technique. The map constraints are learned based on State Vector Machines (SVM) which employ Location Dependent Parameters (LDPs) such as RSSI, ToA and TDoA in order to estimate the room where the user is located. A probabilistic model of user location is hence learned from the user behaviour which can then be converted to geometric map constraints to be input to the RPGA algorithm. The proposed SVM technique shows an error probability of only $1.3 \%$ using 4 RSSIs. The obtained map constraints are then used into RGPA and the Figure 3 compares the positioning accuracy using 4 TOAs with and without map constraints and reveals that using such map constraints enhances the positioning accuracy $(\sim 50 \mathrm{~cm}$ at $80 \%)$.

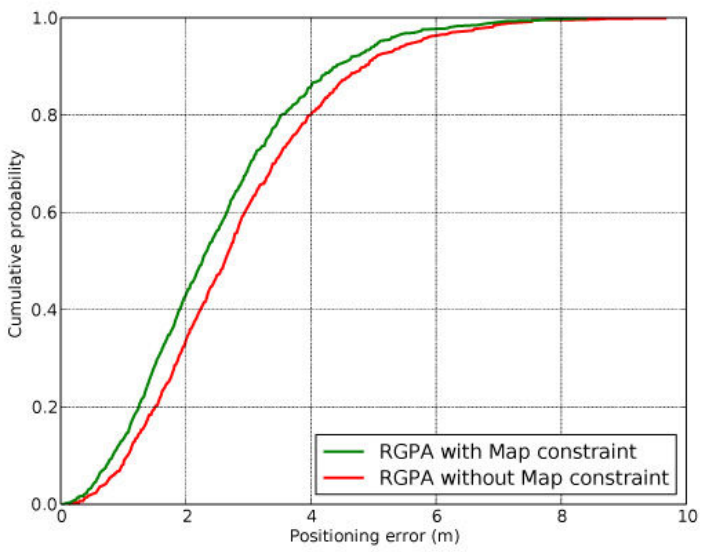

Fig. 3. Map constraints into the RGPA technique

This approach uses map constraints with RGPA (see section II-B.3). In this technique the map constraints are learned based on State Vector Machines (SVM) which employ Location 
Dependent parameters such as RSSI, ToA and TDoA. A probabilistic model of user location is learned from the user behaviour which can then be converted to geometric map constraints to be input to the RPGA algorithm.

\section{RADIO AIDED Positioning}

\section{A. Performance Bounds and Identifiability Conditions for Lo- cation Estimation in NLOS Dynamic Environments}

The non-trivial problem of NLOS localization can be tackled with the aid of geometrical channel and mobility models. This approach results in high performance algorithms but requires a combination of different sources of information, like e.g. angles, delays and Doppler shifts. In order to localize a mobile terminal (MT) in a NLoS environment, the information about the MT location contained in the multipath signal components may be exploited. To that end, we may consider only the first few arriving components and make the assumption that they have bounced only once. The use of this Single Bounce Model (SBM) offers a simple one-to-one mapping between the various LDPs and the coordinates of the MT and the scatterers, for static environments. For dynamic environments, a dynamic-SBM (DSBM), which is the result of the integration of a mobility model with the SBM can be used instead. In [8] we demonstrate the high performance of such methods even in poor scattering environments. On top of that, we compare the algorithms for different scenarios and highlight the superiority of algorithms designed for channels that change dynamically due to the movement of the MT.

\section{B. Adaptation to the environment}

The leading idea here is to exploit the natural complementarity between the infrastructure and the mobile terminal. We propose adaptive knowledge schemes focusing on ways on how to acquire and represent this information allowing easy exploitation for parametric positioning and also how to mitigate uncertainties about the environment and infrastructure.

1) Using Fingerprinting Techniques: Along this section some improvements on the fingerprinting positioning usage are addressed, and also alternative techniques to overcome possible deployments impairments and uncertainties.

One approach fuses together different signal characteristics into a single fingerprinting positioning algorithm for an indoor Wi-Fi environment. For this specific usage, in the learning or training phase a database is informed with a series of data regarding the distance of the user's terminal from the wireless node and the signal characteristics such as SNR, Bit Rate (BR), RSSI etc. Therefore, a database is constructed that consists of a radio and a performance map where each specific location point corresponds to a value of SNR and BR data at specific points which are used for estimating the best location of the user. Preliminary results on this work indicate a mean positioning error of $2.2 \mathrm{~m}$ for a typical indoor environment.

In a second approach the fingerprint location system is assisted by users' historic movements in order to achieve a more accurate localisation system which relies in the users' history inside the building. This is based on the fact that the users' movements tend to have definable patterns imposed by building restrictions. The algorithm developed is based on real RSSI measurements, also taking into account the building layout and constraints. In order to minimise the resources required by exhaustive measurement campaigns during the learning stage an Intermediate Points RSSI estimation algorithm is being proposed (see Figure III-B.1) which allows the collection of fewer RSSI fingerprints and estimates the RSSI values of the intermediate points that measurements have not been performed. A requirement for the algorithm is that there is good knowledge about the building layout in order to extract any building constraints. Results so far indicate that the algorithm performs well achieving a mean error of $2 d B$ compared to measured RSSI values. The next step is to use a positioning algorithm which considers the historic movement of users using the developed fingerprinting database.

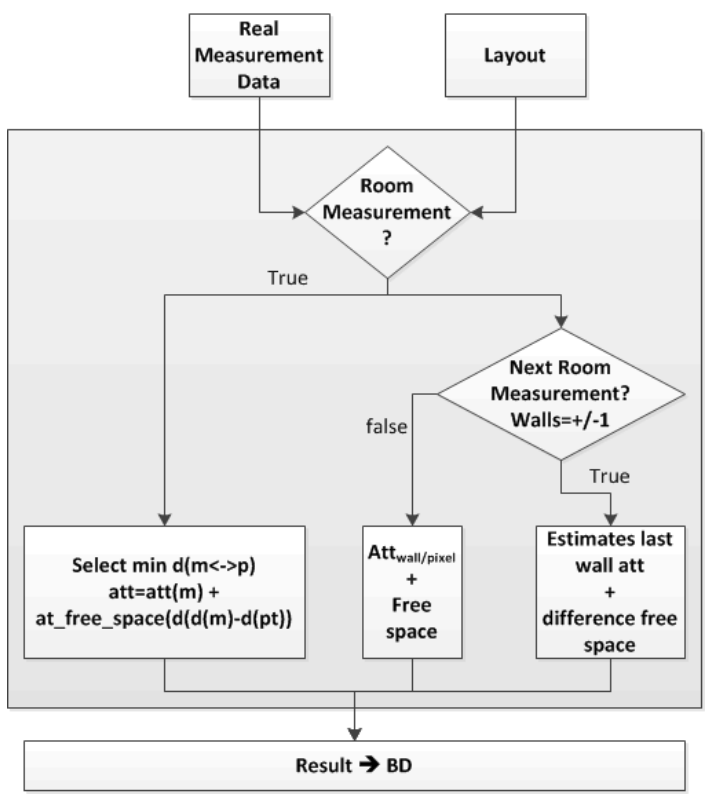

Fig. 4. Block Diagram of the Intermediate's Point Algorithm.

In contrast to the traditional RSS fingerprinting, PDP Fingerprinting (PDP-F) may allow positioning on the basis of a single (Base Station-Mobile Terminal) BS-MT link if the multipath is rich enough (multiple BSs may not be required). Therefore, in a third approach, by exploiting the multipath propagation pattern, PDP Location Finding (LF) creates a signature unique to a given location. Research on the analysis of PDP LF was performed along two directions. Firstly, we focus on a global performance indicator, in the form of Pairwise Error Probability (PEP) [9], [10]. The objective is to determine the probability of error when the channel estimates from the MT-BS link are matched with a wrong entry of the database. Hence position estimation error occurs as a result. We observe that the diversity present in the channel impulse response leads to the same SNR diversity order for PDP-F PEP as for probability of error in digital communications over fading channels. The diversity order corresponds to the number 
of paths in which the PDP differs between the correct and wrong location. In a second investigation, we derive CramérRao bounds (CRBs) for LDPs when they are finite and perform local identifiability analysis under different path amplitude assumptions [11]. We observe that local identifiability of the position vector depends on the number of paths $(L)$ and the modeling of the path amplitudes as well. For the Rayleigh fading case, for the anisotropic modeling (attenuation is a genuine 2D function of position), local identifiability of the position vector can be achieved even for $L=1$. However for the isotropic modeling (attenuation only a function of distance), at least two paths are required ( $L \geq 2$ ) for local identifiability.

Finally, a basic fingerprinting limitation is the time taken and the effort required to train a database and also the fact that the heterogeneity of devices introduces a variable that degrades the positioning performance when the device to be positioned is different from the one that the original measurements have been performed in the learning phase. Therefore, we have studied the use of 3D Ray Tracing (RT) to construct radiomaps for WLAN RSS fingerprint-based positioning, in conjunction with calibration techniques to make the overall process device-independent [12]. We address both challenges by exploiting 3D RT-generated radiomaps and using linear data transformation to match the characteristics of various devices. We evaluate the efficiency of this approach in terms of the time spent to create the radiomap, the amount of data required to calibrate the radiomap for different devices and the positioning error which is compared against the case of using dedicated radiomaps collected with each device. Our performance evaluation indicates that only a small amount of device-specific data are required to reach the same level of positioning accuracy attained with a manually collected radiomap (about $60 \%$ less data collection effort). Thus, our approach is far less laborious compared to traditional radiomap construction. Moreover, the radiomap can be easily updated if the propagation environment changes in the future (e.g. APs are added or removed, furniture or other equipment is relocated, etc.) by running the RT simulator, instead of collecting the radiomap data from scratch.

Ray Tracing accuracy is strongly subject to the accuracy of the input parameters, such as the geometrical (dimensions) and morphological description (electrical parameters of walls) of the buildings and also other uncertainties such as the antenna pattern, location of clutter etc. The work so far involved the implementation of a technique which extracts the electrical parameters of walls using a Vector Network Analyser (VNA). We adopt the multi-pass technique described in [13] which uses the $S_{21}$ parameters obtained using the VNA. A directional antenna is placed in each side of the wall and the complex $S_{21}$ is obtained. The real and imaginary parts of the complex dielectric constant $\epsilon_{r}=\epsilon^{\prime}-j \epsilon^{\prime \prime}$ are calculated through the use of a multiple-pass technique [13] where, an insertion transfer function $H(j \omega)$ that accounts for multiple reflections inside the dielectric slab is required. This insertion function is directly related to the Transmission coefficient $T$ by $T e^{j \beta_{o} d}=H(j \omega)$, where $H(j \omega)$ is the insertion function of the channel, $d$ is the thickness of the material under test and $\beta_{o}=2 \pi f / c$. The transmission coefficient $T$ is equivalent to the measured scattering parameter $S_{21}$. In order to obtain the real and imaginary part of the complex dielectric constant the following expression needs to be solved for $x$ [13].

$$
\left(x+\frac{1}{x}\right) \sinh (x P)+2 \cosh (x P)-\frac{2}{S_{21}}=0
$$

where $x=\sqrt{\epsilon_{r}}, S_{21}=H(j \omega)=e^{-j \omega \tau_{o}}, \tau_{o}=c / d$ and $P=$ $j \beta_{o} d$. This equation is solved numerically for the complex dielectric constant $\epsilon_{r}=\epsilon^{\prime}-j \epsilon^{\prime \prime}$ using two dimensional search algorithms.

2) Using other Techniques: In addition to fingerprinting based techniques for radio aided positioning, we present in this section two contributions both based on the exploitation of RSSI observables. The first presents a technique of ranging based on the RSS-delay profile (RSSDP) instead of simple RSS and the second contribution presents a technique to jointly estimate the path loss model and localising the MTs.

The idea of RSSDP-based ranging (Figure 5) is to estimate the channel impulse response magnitude as a function of delay, possibly average it over fast fading, take the deterministic component as a function of delay and model the random variations due to slow fading and possible residual fast fading. It is then possible to formulate a maximum likelihood estimation problem for the LOS delay (range). In principle this approach should give range identifiability, even in the absence of knowledge about the transmit power, receiver AGC settings, synchronization or a LOS component, due to the range specific curvature of the RSSDP curve over a given delay spread.

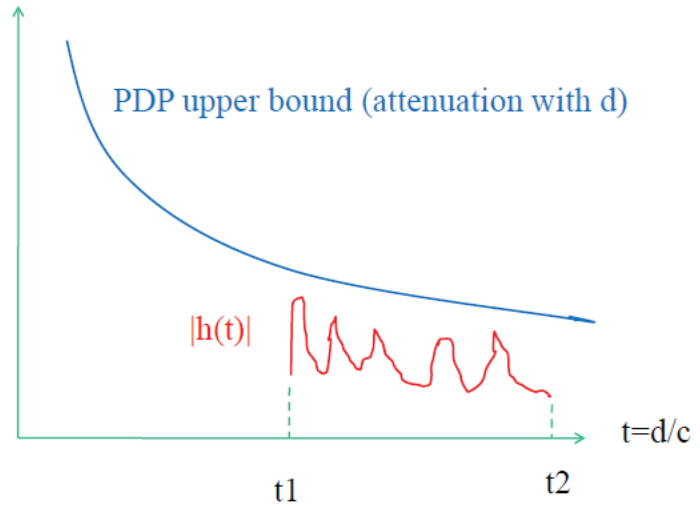

Fig. 5. RSS-Delay Profile/Mask (RSSDM) based Range Estimation.

For the joint localisation and path loss learning we propose technique based on State Vector Machine (SVM) aiming to continuously learn and update the path loss model jointly with the localization task. The assumed scenario is a situation where each BS is collecting RSSI measurements from different MTs moving in its coverage and use these information to update its path loss model and feedback to the MTs in order to be localized. Both one-slope and multi-slope models are considered. The used model in all this work is the log-normal shadowing model which is defined by Equation (3) 


$$
E_{r}(d B)=E_{0}-10 n_{p} \log _{10}\left(\frac{d}{d_{0}}\right)+X_{s h}
$$

where $E_{r}$ is the received energy, $E_{0}$ is the received energy at $d_{0}$ which is taken equal to 1 meter, $n_{p}$ is the propagation constant, and $X_{s h}$ is a zero-mean Gaussian random variable with a standard deviation $\sigma_{s h}$ which models the shadowing.

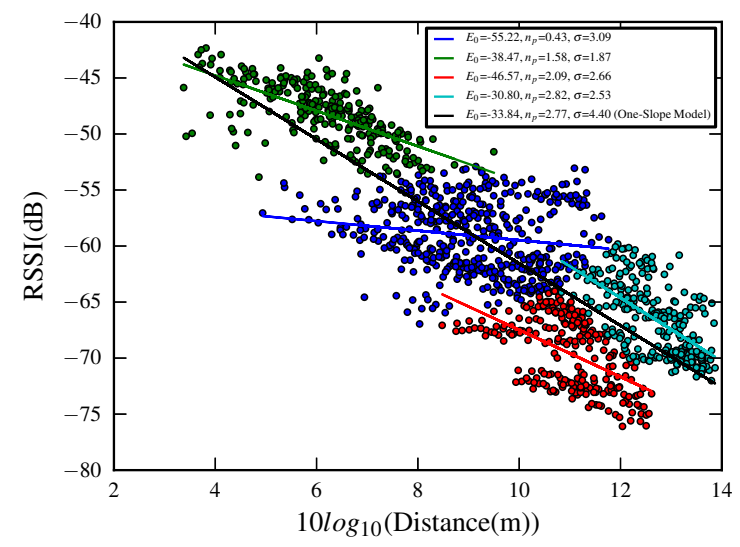

Fig. 6. Multi-slope Model vs. One-Slope Model

Using distance and RSSI information, the SVM technique classifies the points in different clusters which are homogeneous and have lower variance and dispersion than the oneslope model. For each new obtained data, the BSs apply the SVM technique in order to classify this data and then update the multi-slope model which then represents better the radio channel and its fluctuations. Figure 6 shows an example of multi-slope model obtained on MA-UWB measurements. A comparison between ranging performances of one-slope and multi-slope models indicates an interesting enhancement which reaches 1.4 meters at $80 \%$.

\section{CONCLUSION}

This paper summarizes the ongoing activities in the WHERE-2 Project on the topic of location information extraction and how this information can be used in positioning. More particularly we present preliminary results on the exploitation of non-radio and radio location specific parameters and how these can be used into improved positioning algorithms.

Regarding non-radio context the exploitation of information received from inertial sensors as well as information which can be extracted from environment maps has been studied. Different approaches for performing Kalman filtering have been identified and a particular tracking filter embodiment was designed to enable the loose fusion between non-collocated radios and inertial units. Also an application which combines ankle-mounted inertial sensors with an existing UWB platform has been presented. Finally, extracted map-related information is being used into two different fingerprinting techniques.

More advanced positioning algorithms which utilize location-specific radio information are being proposed. One approach deals with performance bounds and identifiability conditions for positioning in NLOS environments by exploiting multipath information. Several research activities have also been carried out addressing the improvement and usage of radio aided positioning systems through fingerprinting by exploiting and fusing the available context and also ways to overcome various uncertainties about the environment. Also we deal with the problem when training and using a fingerprinting database using different devices. Finally other techniques which exploit RSSDP information and RSSI observables for path loss learning are being presented.

The results and techniques presented in this paper are part of an on-going research and more definite solutions are expected to be presented in the future based on the encouraging results presented here. More information can be found in Deliverable D2.2 of the WHERE-2 Project [14].

\section{ACKNOWLEDGMENT}

This work has been performed in the framework of the ICT248894 WHERE2 project funded by the European Union.

\section{REFERENCES}

[1] [Online]. Available: http://www.x-io.co.uk/node/8

[2] [Online]. Available:
io.co.uk/res/doc/madgwick_internal_report.pdf

http://www.x-

S. Bensaid and D. Slock, "'Comparison of Various Approaches for Joint Wiener/Kalman Filtering and Parameter Estimation with Application to BASS"," in Proc. IEEE Asilomar Conf. on Signals, Systems and Computers, Pacific Grove, CA, USA, Nov. 2011.

[4] J. Youssef, B. Denis, C. Godin, and S. Lesecq, "Loosely-coupled iruwb handset and ankle-mounted inertial unit for indoor navigation," in Ultra-Wideband (ICUWB), 2011 IEEE International Conference on, sept. 2011, pp. $160-164$.

[5] —, "New TOA Estimators within Energy-based Receivers under Realistic UWB Channel Statistics," in IEEE VTC-Spring 10, Taipei, Taiwan, May 2010.

[6] — "Reducing the Complexity Order of Position Estimator with Combined Radiolocation Measurements," in WPNC 2009, Hanover, Germany, March 2009.

[7] T. Roos, P. Myllymaki, H. Tirri, P. Misikangas, and J. Sievanen, "A probabilistic approach to WLAN user location estimation," International Journal of Wireless Information Networks, vol. 9, no. 3, pp. 155-164, July 2002.

[8] K. Papakonstantinou and D. Slock, “"Performance bounds and identifiability conditions for location estimation in NLOS dynamic environments"," in Proc. IEEE-SP Workshop on Statistical Signal Processing (SSP), Nice, France, June 2011.

[9] T. Oktem and D. Slock, "'Pairwise Error Probability Analysis for Power Delay Profile Fingerprinting based Localization"," in Proc. IEEE Vehicular Technology Conf. (VTCspring), Budapest, Hungary, May 2011.

[10] D. Slock, "'Diversity Aspects of Power Delay Profile based Location Fingerprinting"," in In Proc. IEEE-SP Int'l Conf. on Acoustics, Speech and Signal Processing(ICASSP), Kyoto, Japan, March 2012.

[11] T. Oktem and D. Slock, "'Cramer-Rao Bounds for Power Delay Profile Fingerprinting based Positioning "," in Proc. IEEE-SP Int'l Conf. on Acoustics, Speech and Signal Processing (ICASSP), Prague, Czech Republic, May 2011.

[12] M. Raspopoulos, C. Laoudias, L. Kanaris, A. K. andC. G. Panayiotou, and S. Stavrou, "3d ray tracing for device-independent fingerprint-based positioning in wlans," in 9th Workshop on Positioning, Navigation and Communication 2012 (WPNC'12), Dresden, Germany, March 2012.

[13] A. H. Muquibel and A. S. Jazi, "A new formulation for characterisation of materials based on insertiontransfer function," IEEE Trans. Microw. Theory and Techniques, 2003.

[14] M. Raspopoulos, S. Stavrou, B. Denis, D. Slock, J. Stephan, M. Laaraiedh, J. D. G. Agapiou, B. Uguen, and L. D. Celis, "Intermediate: Location information extraction," Deliverable D2.2 WHERE-2 Project, March 2012. 